\title{
Examining the Impact of Refractory Myasthenia Gravis on Healthcare Resource Utilization in the United States: Analysis of a Myasthenia Gravis Foundation of America Patient Registry Sample
}

\author{
Haichang Xin ${ }^{\mathrm{a}}$ \\ Linda A. Harris ${ }^{b}$ \\ Inmaculada B. Aban ${ }^{\mathrm{C}}$ \\ Gary Cutter ${ }^{\mathrm{C}}$ \\ aDepartment of Health Care Organization \\ and Policy, University of Alabama \\ at Birmingham, Birmingham, AL, USA \\ ${ }^{b}$ Alexion Pharmaceuticals, Inc., Boston, \\ MA, USA \\ 'Department of Biostatistics, \\ University of Alabama at Birmingham, \\ Birmingham, AL, USA
}

\begin{abstract}
Background and Purpose Patients with refractory myasthenia gravis (MG) experience ongoing disease burden that might be reflected in their healthcare utilization. Here we examine the impact of refractory MG on healthcare utilization.

Methods The 825 included participants were aged 18-64 years, enrolled in the Myasthenia Gravis Foundation of America Patient Registry between July 2013 and February 2018, and had been diagnosed with $M G \geq 2$ years previously.

Results Participants comprised 76 (9.2\%) with refractory MG and 749 (90.8\%) with nonrefractory MG. During the 6 months before enrollment, participants with refractory MG were significantly more likely than those with nonrefractory MG to have experienced at least one exacerbation $[67.1 \%$ vs. $52.0 \%$, respectively, $p=0.01$; odds ratio $(\mathrm{OR})=1.882,95 \%$ confidence interval $(\mathrm{CI})=1.141-3.104]$, visited an emergency room at least once [ $43.4 \%$ vs. $27.1 \%, p<0.01$; $\mathrm{OR}=2.065,95 \% \mathrm{CI}=1.276-3.343$ ], been hospitalized overnight at least once $(32.9 \%$ vs. $20.5 \%$, $p=0.01 ; \mathrm{OR}=1.900,95 \% \mathrm{CI}=1.140-3.165$ ), ever been admitted to an intensive care unit (ICU) (61.8\% vs. $33.4 \%, p<0.01$; OR=3.233, $95 \% \mathrm{CI}=1.985-5.266)$, or ever required a feeding tube ( $21.1 \%$ vs. $9.1 \%, p<0.01 ; \mathrm{OR}=2.671,95 \% \mathrm{CI}=1.457-4.896)$. A total of $75.8 \%$ younger females with refractory disease ( $<51$ years, $n=33$ ) experienced at least one exacerbation, $69.7 \%$ had been admitted to an ICU, and $30.3 \%$ had required a feeding tube. For older females with refractory disease ( $\geq 51$ years, $n=33$ ), $60.6 \%, 54.6 \%$, and $6.1 \%$ experienced these outcomes, respectively (between-group differences were not significant).

Conclusions Refractory MG is associated with higher disease burden and healthcare utilization than nonrefractory MG.
\end{abstract}

Key Words myasthenia gravis, refractory disease, disease exacerbations, healthcare resource utilization.
Received December 26, 2018

Revised March 8, 2019

Accepted March 12, 2019

\section{Correspondence}

Inmaculada B. Aban, MS, PhD

Department of Biostatistics,

University of Alabama at Birmingham, 1665 University Blvd, Birmingham,

AL 35233, USA

Tel +1-205-934-2732

Fax +1-205-975-2541

E-mail caban@uab.edu

\section{INTRODUCTION}

Myasthenia gravis (MG) is a chronic rare autoimmune disease characterized by severe muscle weakness and caused by the inhibition of neuromuscular transmission due to the binding of autoantibodies at the neuromuscular junction and subsequent complementmediated destruction of the end-plate region. The prevalence of MG has been estimated as ranging from 107 to 278 per million. ${ }^{1-5} \mathrm{MG}$ often first manifests as weakness of ocular muscles (ocular MG), but approximately $70-80 \%$ of patients eventually manifest generalized disease that affects other muscles including in the face, neck, hand, and/or limb..$^{6-9}$ This generalized muscle weakness can lead to symptoms that include slurred speech, difficulty swal-

(a) This is an Open Access article distributed under the terms of the Creative Commons Attribution Non-Commercial License (https://creativecommons.org/licenses/by-nc/4.0) which permits unrestricted non-commercial use, distribution, and reproduction in any medium, provided the original work is properly cited. 
lowing, extreme fatigue, and weakness of the upper and lower extremities, which together can have a considerable impact on the ability to perform the activities of daily living (ADL). ${ }^{10-12}$ Reportedly, $15-20 \%$ of patients experience severe weakness of the respiratory muscles that can lead to life-threatening respiratory failure (myasthenic crisis) and result in the need for intubation and mechanical ventilation. ${ }^{13,14}$

Acetylcholinesterase inhibitors, corticosteroids, or steroidsparing immunosuppressive therapies (ISTs) are effective against most or all of the disease symptoms for many patients with MG. ${ }^{15,16}$ However, approximately $15 \%$ of patients do not exhibit adequate symptom control or cannot tolerate these treatments, and are therefore considered to have refractory MG. ${ }^{17}$ The criteria that are frequently used to define refractory disease include 1) failure to respond to adequate doses of conventional therapies, 2) inability to reduce IST use without clinical relapse or a need for ongoing rescue therapy including plasma exchange (PLEX) or intravenous immunoglobulin G (IVIg), 3) severe and/or intolerable side effects to IST, 4) comorbid conditions restricting the use of conventional therapies, and 5) frequent myasthenic crises. ${ }^{13,18} \mathrm{~A}$ large number of patients with refractory MG spend many years receiving a range of traditional treatments in attempts to achieve disease control, before progressing to more-intensive treatments including chronic IVIg or PLEX. ${ }^{16}$ The health-related quality of life of patients with MG is negatively impacted when adequate symptom control cannot be achieved and by the possible burden of MG-treatment side effects. ${ }^{19,20}$

In light of the persistent clinical burden experienced by patients with refractory $\mathrm{MG}$ as a result of poor symptom control and increased risk of MG exacerbations, it is reasonable to assume that patients with refractory MG use more healthcare resources than those with nonrefractory disease. Data from a United States (US) claims database have shown that compared with those with nonrefractory MG, patients with refractory MG who experience more myasthenic exacerbations (including crises) visit the emergency room (ER) more often and have more inpatient hospitalizations. ${ }^{21}$ Similar findings have been reported for a Japanese study analyzing anonymized patient health records. ${ }^{22}$

The present study used patient-reported data to further examine the impact of refractory MG on healthcare resource utilization (HRU) in the USA and to corroborate the findings reported based on US claims data.

\section{METHODS}

\section{Data source}

The Myasthenia Gravis Foundation of America (MGFA) Patient Registry is an active database of individuals with MG that has been developed for use in research and treatment development. This registry is open to adults who are $\geq 18$ years of age and living in the USA. Participants enter the registry by completing an online enrollment survey that comprises approximately 200 questions covering categories including participant demographics, MG history, comorbidities, past and current therapies, family history of MG, functional status [assessed using the myasthenia gravis activities of daily living scale (MG-ADL)], lifestyle, and HRU. The MG-ADL is a validated eight-item, participant-reported outcome measure that was developed to assess MG symptoms and their functional impact; the MG-ADL total score ranges from 0 to 24 , with a higher score indicating worse impact on ADL. ${ }^{10,23}$

The present study included data from enrollment surveys completed between July 2013 (when the MGFA Patient Registry was instigated) and February 2018. Participants receive a shorter online survey to complete every 6 months after enrollment, but no data from these post enrollment surveys were included in the present study. Data were de-identified for research use, and consent for participation was provided by participants electronically at registration before they completed the enrollment survey. The study was approved by the Institutional Review Board of the University of Alabama at Birmingham, Birmingham, AL, USA (IRB No. 130401004).

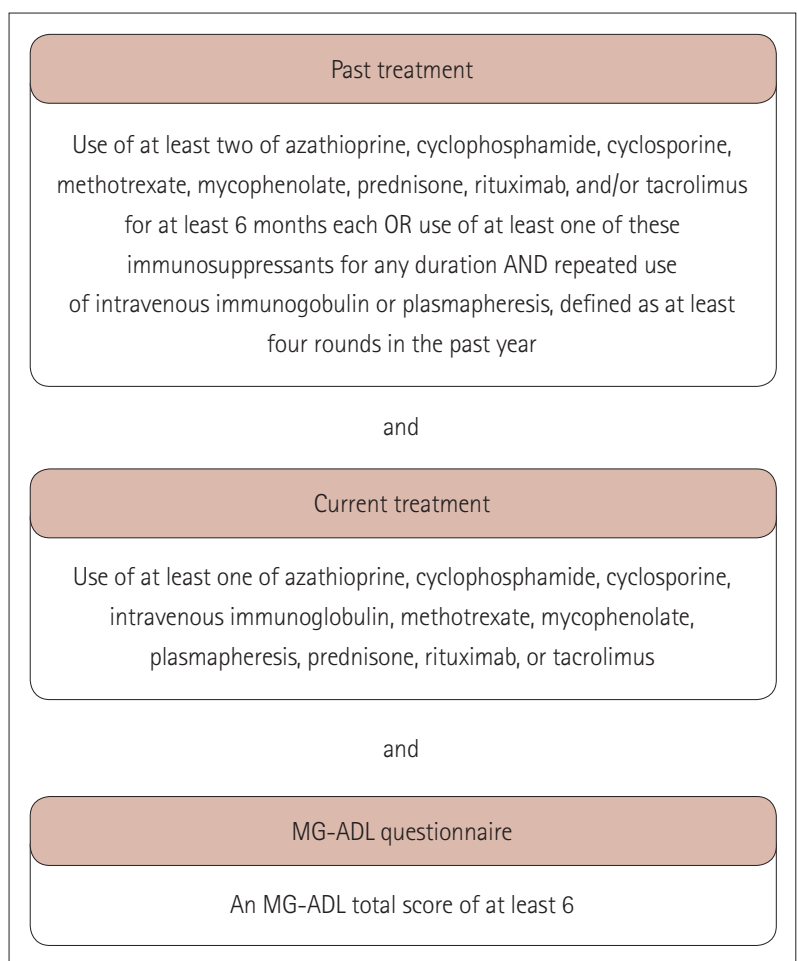

Fig. 1. Criteria used for classifying refractory MG. MG-ADL: myasthenia gravis activities of daily living scale. 
Impact of Refractory MG on Healthcare Utilization

Table 1. Summary of the Myasthenia Gravis Foundation of America patient registry enrollment questions used in the study

\begin{tabular}{|c|c|}
\hline Question & Predefined response categories \\
\hline Your date of birth & MM/DD/YYYY \\
\hline Your gender & Male; female \\
\hline Are you of Hispanic, Latino, or Spanish origin? & Yes; no \\
\hline What is your current marital status? & $\begin{array}{l}\text { Never married; married; divorced; widowed; separated; cohabitation/domestic } \\
\text { partner }\end{array}$ \\
\hline With whom do you currently live? & $\begin{array}{l}\text { Alone; spouse/partner; sibling; children; parent; other relative; domestic help; } \\
\text { friend/companion; caregiver; in nursing or sheltered/assisted home }\end{array}$ \\
\hline Please select your highest level of formal education completed & $\begin{array}{l}\text { Less than high school; high-school degree/GED; associate's degree; technical } \\
\text { degree; bachelor's degree; postgraduate degree (master's or doctorate) }\end{array}$ \\
\hline $\begin{array}{l}\text { Based on the definition above, }{ }^{*} \text { have you had an exacerbation } \\
\text { in the last } 6 \text { months? }\end{array}$ & Yes; no; unsure \\
\hline If yes, how many exacerbations have you had? & $1 ; 2 ; 3 ; 4 ; 5$ or more \\
\hline $\begin{array}{l}\text { In the last } 6 \text { months, how many times did you visit } \\
\text { an emergency room? }\end{array}$ & $0 ; 1 ; 2 ; 3 ; 4 ; 5 ; 6 ; 7 ; 8 ; 9 ; 10 ; 11 ; 12 ; 13$ or more; unknown \\
\hline $\begin{array}{l}\text { In the last } 6 \text { months, were you admitted overnight to a healthcare } \\
\text { institution? (including general hospital, rehabilitation hospital, or } \\
\text { nursing home) }\end{array}$ & Yes; no \\
\hline \multirow[t]{4}{*}{ If yes, what were the reasons? } & Exacerbation or worsening of $\mathrm{MG}$ \\
\hline & Rehabilitation \\
\hline & Other MG-related problem \\
\hline & Non-MG-related problem \\
\hline Have you ever been admitted to an intensive care unit for your MG? & Yes; no; unsure \\
\hline Have you ever required a feeding tube? & Yes; no; unsure \\
\hline
\end{tabular}

Reproduced with permission from the MGFA.

*Definition of an exacerbation of MG: development of new symptoms or worsening of existing symptoms that lasted $>7$ days and occurred $\geq 30$ days after the last exacerbation. In an exacerbation, MG symptoms generally worsen over a period of days to weeks. They then improve over several weeks or months, usually with specific treatment. An exacerbation can be associated with several different symptoms that worsen simultaneously.

GED: general equivalency diploma, MG: myasthenia gravis.

\section{Participants}

Participants in the MGFA Patient Registry were included in this study if they were aged $\geq 18$ years and $<65$ years. This age restriction was applied in order to minimize the impact on HRU of comorbidities and mortality that may be experienced by individuals aged $\geq 65$ years. Moreover, US residents aged $\geq 65$ years are eligible for Medicare, a government-funded hospital insurance cover, which may increase the number of times these individuals visit a healthcare establishment for MG-related treatment.

Participants were included in this study if they reported having been diagnosed by their doctor as having MG for $\geq 2$ years before completing the enrollment survey. This time requirement was used to ensure that adequate time had elapsed between the diagnosis of MG and inclusion in the study for the presence of refractory disease (defined as no or inadequate response to therapy) to be determined. Participants were classified as having refractory MG based on their MG treatment history [previous use of at least two ISTs (azathioprine, cyclophosphamide, cyclosporine, methotrexate, mycophenolate, prednisone, rituximab, and/or tacrolimus) for at least 6 months each, or previous use of at least one of these ISTs for any duration and repeated use of IVIg or PLEX (at least four rounds in the previous year)], current use of at least one MG treatment, and having an MG-ADL total score of at least 6 (Fig. 1). Participants who did not meet the previous or current treatment criteria for refractory MG were considered to have nonrefractory $\mathrm{MG}$ regardless of their MGADL score. Participants for whom there were no data available to enable classification as refractory or nonrefractory were excluded from this analysis.

\section{Study measures}

The primary outcome measures were the proportions of participants who had experienced at least one exacerbation, visited an ER at least once, and/or been hospitalized overnight at least once during the 6 months before enrollment, and the proportions who had ever been admitted to an intensive care unit (ICU) for reasons related to MG and/or required a feeding tube. The numbers of MG exacerbations and ER visits 


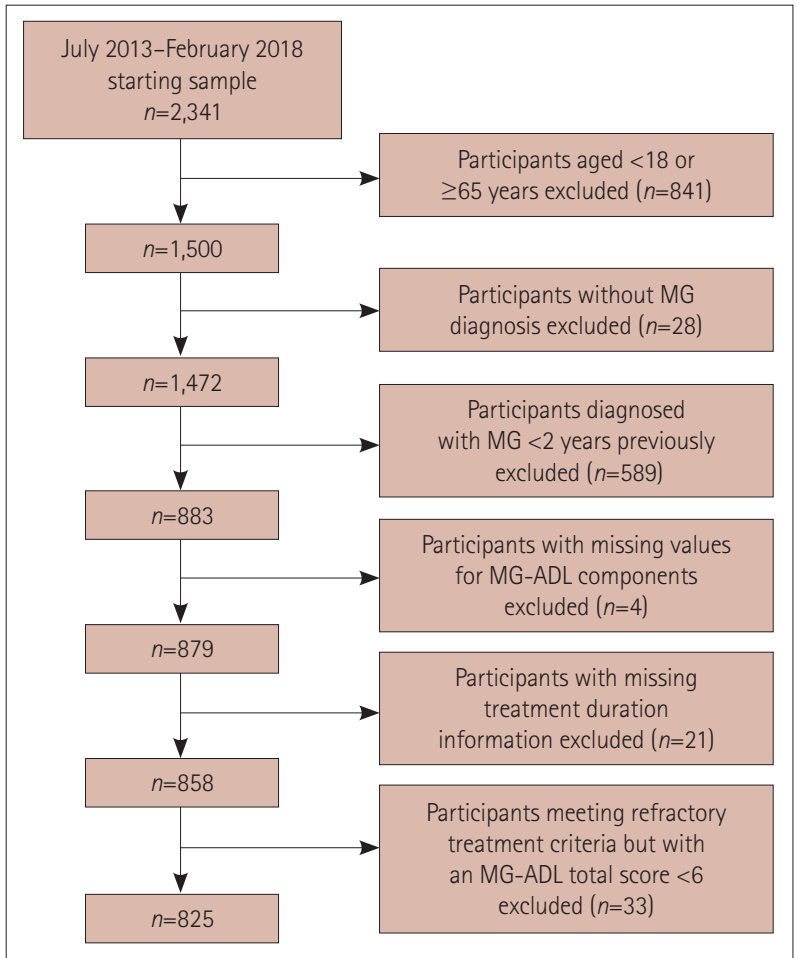

Fig. 2. Flow chart showing selection process for the study sample. MG-ADL: myasthenia gravis activities of daily living scale.

during the 6 months before enrollment were also recorded. The numbers of exacerbations and ER visits were each categorized into $0-3$ and $\geq 4$ in order to distinguish between lower and higher disease burdens. The enrollment survey defined an exacerbation of MG as the development of new symptoms or the worsening of existing symptoms that lasted $>7$ days and occurred $\geq 30$ days after the last exacerbation. Other variables of interest due to their potential association with HRU included age, gender, ethnicity, living arrangement, marital status, and education level. The questions relating to the demographic and HRU data extracted for this study are listed in Table 1.

\section{Statistical analyses}

Summary statistics were calculated for all study variables. Bivariate analyses were used to compare demographic, exacerbation, and HRU data for the refractory- and nonrefractoryMG groups and to compare exacerbation and HRU data between younger and older female participants with refractory MG. $\chi^{2}$ tests (or Fisher's exact tests for small samples) were used for categorical variables, and $t$-tests were used for continuous variables.

Based on the median age of the study population (51 years), participants were classified as being younger if they were aged $<51$ years and older if they were aged $\geq 51$ years. Analyses were based on fitting a logistic model for binary out- comes, except when analyzing the numbers of exacerbations and ER visits, for which proportional-odds models for ordinal outcomes were fitted. Unadjusted and adjusted models were fitted to examine the effects of covariates on the association between refractory status and the exacerbation and HRU outcomes. The covariates included were the living arrangement, marital status, age (with 51 years as the cutoff), gender, ethnicity, and education level. Subgroup analyses by age within the refractory group were applied to female participants only, since an equivalent subgroup analysis with male participants only was not possible due to the small number of males with refractory disease $(n=10)$. No unknown or missing-value categories for any of the outcomes were included in the statistical analyses. Probability values of $p<0.05$ were deemed significant. Analyses were performed using SAS $^{\circledR}$ (version 9.4, SAS Institute, Cary, NC, USA).

\section{RESULTS}

The flow of participants through the sample selection process is summarized in Fig. 2. The 2,341 participants in the MGFA Patient Registry included 825 participants who met the inclusion criteria of this study: 76 (9.2\%) were classified as having refractory MG based on the criteria defined for this study, and 749 participants $(90.8 \%)$ were classified as having nonrefractory MG (Table 2). The bivariate analyses revealed no statistically significant differences between the refractory and nonrefractory groups with regard to demographic data including age and gender (Table 2).

The bivariate analyses revealed statistically significant associations between refractory MG and the outcomes for exacerbations and HRU. During the 6 months before enrollment, a significantly higher proportion of participants with refractory MG than those with nonrefractory MG had experienced at least one MG exacerbation, had visited an ER at least once, or had been hospitalized at least once (Fig. 3A). Furthermore, a higher proportion of participants with refractory MG than those with nonrefractory MG had experienced at least four exacerbations or had visited an ER at least four times (Fig. 3B). Compared with participants with nonrefractory MG, a significantly higher proportion of participants with refractory MG had been admitted to an ICU at any time for reasons associated with $\mathrm{MG}$ or had previously required a feeding tube (Fig. 3A).

Unadjusted regression analyses confirmed that participants with refractory $M G$ were significantly more likely than those with nonrefractory MG to have experienced at least one MG exacerbation, visited an ER at least once, been hospitalized at least once, been admitted to an ICU at any time for reasons associated with $\mathrm{MG}$, or previously required a 
Table 2. Participant demographics

\begin{tabular}{|c|c|c|c|}
\hline Characteristic & Refractory MG $(n=76)$ & Nonrefractory MG $(n=749)$ & $p$ \\
\hline Age at time of survey completion, years & $48.0 \pm 11.4$ & $49.3 \pm 11.1$ & 0.31 \\
\hline Age group, years & & & 0.51 \\
\hline $18-24$ & $1(1.3)$ & $14(1.9)$ & \\
\hline $25-34$ & $12(15.8)$ & $81(10.8)$ & \\
\hline $35-44$ & $19(25.0)$ & $156(20.8)$ & \\
\hline $45-54$ & $17(22.4)$ & $216(28.8)$ & \\
\hline $55-64$ & $27(35.5)$ & $282(37.7)$ & \\
\hline Female & $66(86.8)$ & $590(78.8)$ & 0.10 \\
\hline Ethnicity & & & 0.47 \\
\hline Hispanic/Latino/Spanish & $6(7.9)$ & $43(5.7)$ & \\
\hline Not Hispanic/Latino/Spanish & $70(92.1)$ & $697(93.1)$ & \\
\hline Missing/unknown & 0 & $9(1.2)$ & \\
\hline Marital status* & & & 0.55 \\
\hline Married or cohabiting/domestic partner & $47(61.8)$ & $477(63.7)$ & \\
\hline Unmarried or not cohabiting/no domestic partner & $28(36.8)$ & $245(32.7)$ & \\
\hline Missing/unknown & $1(1.3)$ & $27(3.6)$ & \\
\hline Living arrangement $^{+}$ & & & 0.39 \\
\hline With others & 68 (89.5) & $621(82.9)$ & \\
\hline Alone & $8(10.5)$ & $102(13.6)$ & \\
\hline Missing/unknown & 0 & $26(3.5)$ & \\
\hline Education level & & & 0.37 \\
\hline Not graduated from high school & $3(3.9)$ & $10(1.3)$ & \\
\hline High-school degree/GED & $23(30.3)$ & $200(26.7)$ & \\
\hline Associate's degree & $13(17.1)$ & $117(15.6)$ & \\
\hline Technical degree & $6(7.9)$ & $44(5.9)$ & \\
\hline Bachelor's degree & $14(18.4)$ & $194(25.9)$ & \\
\hline Postgraduate degree & $17(22.4)$ & $177(23.6)$ & \\
\hline Missing/unknown & 0 & $7(0.9)$ & \\
\hline MG-ADL total score & $9.6 \pm 2.7$ & $6.7 \pm 4.0$ & $<0.01$ \\
\hline
\end{tabular}

Data are mean \pm standard deviation or $n(\%)$ values. Frequency or mean values of study variables were compared between participants with refractory and nonrefractory MG using $\chi^{2}$ tests for categorical variables and $t$-tests for continuous variables. $p$ values were calculated after excluding participants with unknown/missing data.

*Six predefined options, which were simplified into married or unmarried, 'Ten predefined options, which were simplified into living alone or with others.

GED: general equivalency diploma, MG-ADL: myasthenia gravis activities of daily living scale.

feeding tube (Fig. 4). Similarly, compared with participants with nonrefractory MG, participants with refractory MG were significantly more likely to have experienced at least four exacerbations or had visited an ER at least four times (Fig. 4). After adjusting for other demographic variables such as age, gender, ethnicity, marital status, and education level, the estimated odds ratios were similar to the unadjusted odds ratios across all outcome measures (data not shown).

Among the 66 female participants with refractory MG, a significantly higher proportion of the younger ones $(n=33)$ than older ones $(n=33)$ had previously required a feeding tube (Fig. 5A). A total of $75.8 \%$ of younger female participants with refractory disease experienced at least one exacerbation and $69.7 \%$ had experienced an ICU admission (Fig. 5A). For older female participants with refractory disease, $60.6 \%$ and $54.6 \%$ had experienced at least one exacerbation or ICU admission, respectively (Fig. 5A). Just over a fifth of younger female participants (21.2\%) experienced at least four exacerbations and 6.1\% visited an ER at least four times (Fig. 5B). For older female participants, $18.2 \%$ experienced at least four exacerbations and 9.1\% visited an ER at least four times (Fig. 5B). The between-group differences were not significant (Fig. 5).

\section{DISCUSSION}

This study was designed to examine the impact of refractory 

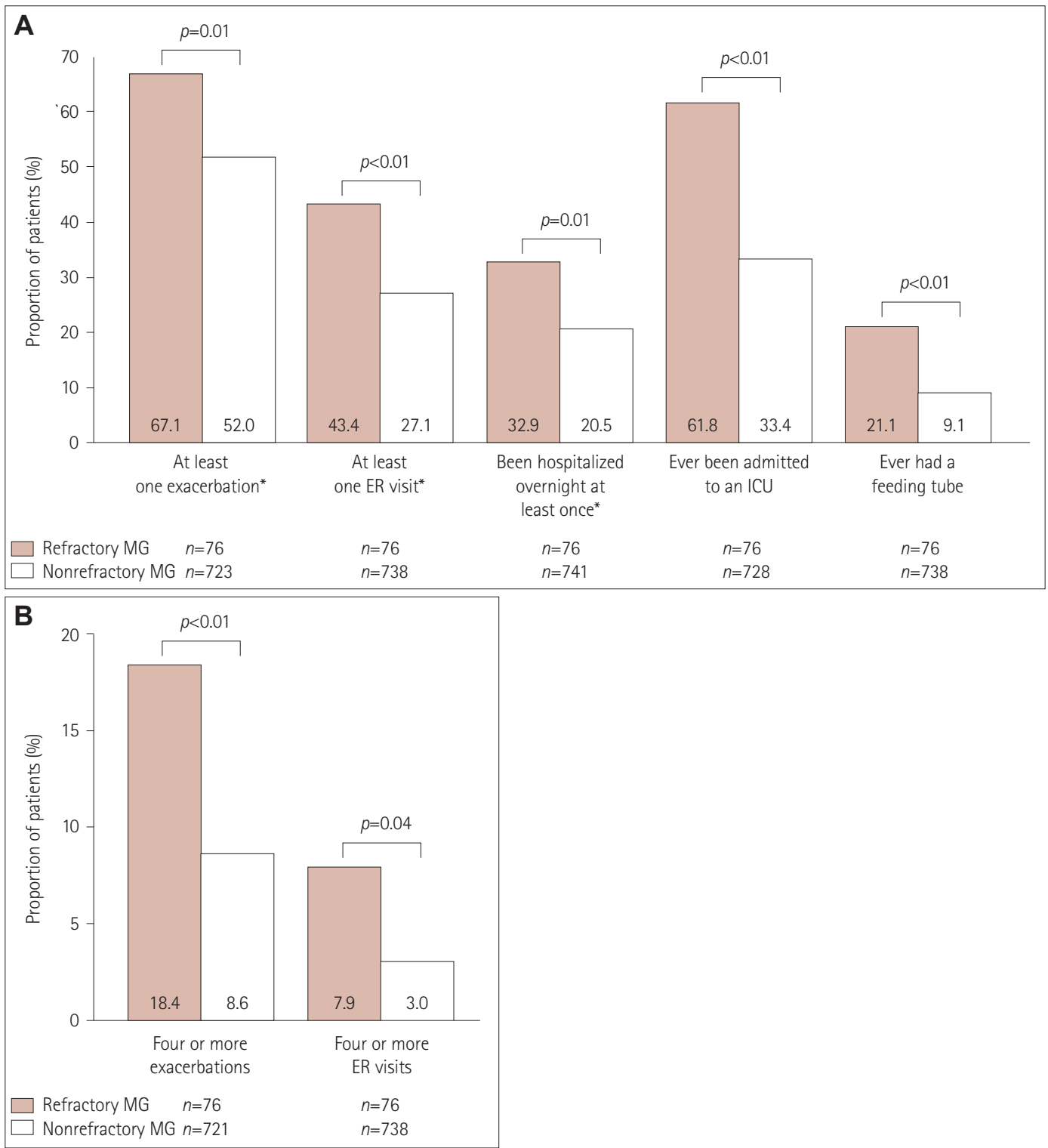

Fig. 3. Exacerbations in and healthcare resource utilization by participants with refractory $M G$ and nonrefractory MG (A) and numbers of exacerbations and ER visits during the 6 months before enrollment in participants with refractory and nonrefractory MG (B). Frequencies of study variables were compared between participants with refractory and nonrefractory MG using $\chi^{2}$ tests for categorical variables and the number of exacerbations, and Fisher's exact test for the number of ER visits. $p$ values are for comparisons between the refractory- and nonrefractory-MG groups, and were calculated after excluding data from participants with unknown/missing data. *During the 6 months before enrollment. ER: emergency room, ICU: intensive care unit, MG: myasthenia gravis.

MG compared with nonrefractory MG on the number of exacerbations and HRU in the USA using patient-reported data extracted from the MGFA Patient Registry. Our findings from the bivariate and unadjusted regression analyses support the hypothesis that patients with refractory MG have more exacerbations and utilize more healthcare resources than those with nonrefractory MG.

There is currently no widely accepted definition of refractory MG. ${ }^{18}$ In the present study we considered a patient to have refractory MG if they experienced persistent symptoms after receiving at least two ISTs or at least one IST and repeated use of IVIg or PLEX in the past, and were currently receiving treatment and still had an MG-ADL total score of at least 6 (Fig. 1). This conservative definition is similar to those used in other studies. ${ }^{18,24}$

Participants with refractory MG accounted for $9.2 \%$ of the individuals in this study, which is similar to the proportions included in other studies. ${ }^{17,21}$ The participant demographics, including age and gender, were not significantly different between the refractory and nonrefractory MG groups. The high 


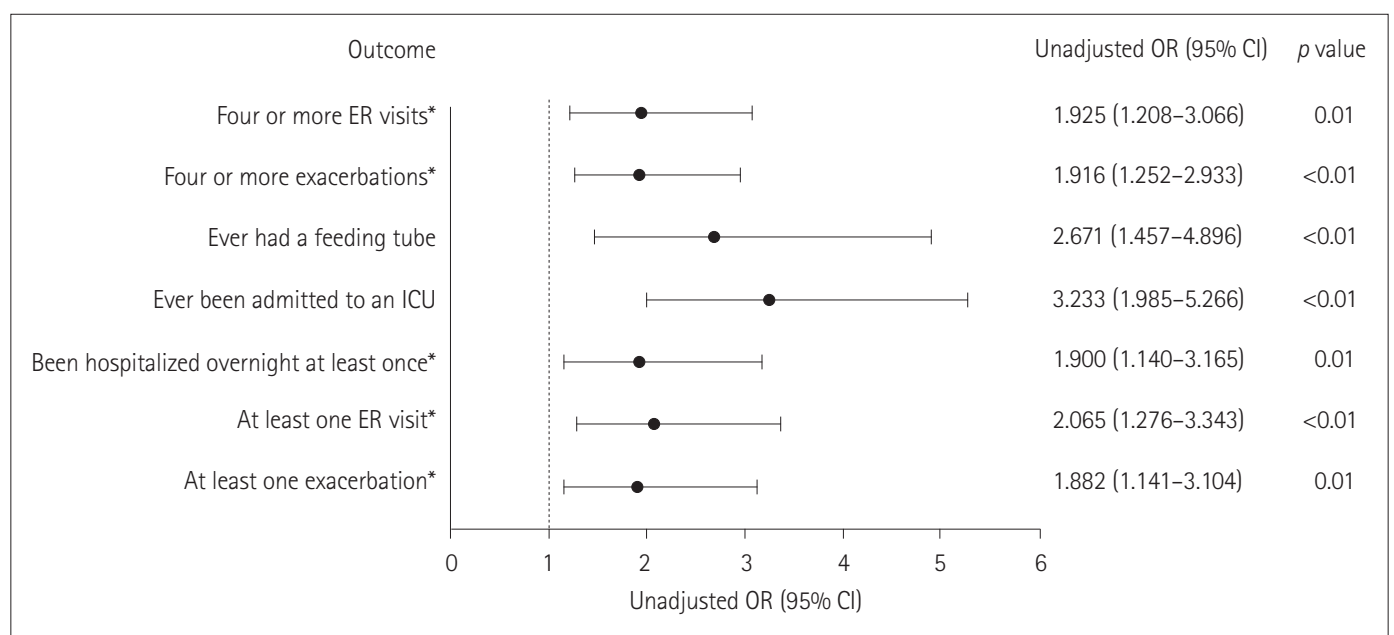

Fig. 4. Unadjusted ordinal logistic model examining the impact of refractory MG on exacerbations and healthcare resource utilization. ${ }^{*} D u r i n g$ the 6 months before enrollment. Analyses were based on fitting logistic models for binary outcomes except for when analyzing the numbers of exacerbations and ER visits, for which proportional-odds models for ordinal outcomes were fitted. Cl: confidence interval, ER: emergency room, ICU: intensive care unit, MG: myasthenia gravis, OR: odds ratio.

proportion of females in the study population (80\%) may have resulted from excluding participants aged $\geq 65$ years, because females have an earlier mean age at MG onset ${ }^{6,25}$ and so they will constitute a higher proportion of a young population than males.

Considering the study population as a whole, over half of the participants (53.4\%) reported experiencing an exacerbation and almost one-third (28.6\%) had visited an ER at least once during the 6 months before enrollment. The findings of this study highlight not only the burden of disease experienced by patients with refractory MG, but also the impact of MG on patients with nonrefractory MG. The current study corroborates the findings of previous studies that examined the impact of refractory MG on HRU based on claims databases and patient health records. ${ }^{21,22}$ The present study found that more participants with MG refractory to conventional therapies had experienced exacerbations, ER visits, and overnight hospitalizations during the 6 months before enrollment. Additionally, participants with refractory-MG were more likely than those with nonrefractory MG to have ever been admitted to an ICU for reasons associated with MG, or to have required a feeding tube.

MG is associated with an increased risk of comorbidities including dysthyroidism, dyslipidemia, diabetes mellitus, and other autoimmune diseases (e.g., autoimmune thyroiditis, systemic lupus erythematosus, and rheumatoid arthritis), and the presence of a comorbid disease has been associated with more-frequent myasthenic crises and ER visits. ${ }^{26-28}$ It might be expected that the occurrence of comorbiditiesand therefore HRU—-would increase with age. In the present study, the proportions of younger and older female par- ticipants with refractory MG who visited an ER at least four times were $6.1 \%$ and $9.1 \%$ respectively ( $p=0.64$ ), although it should be noted that this result was not statistically significant and that participants in this study were aged $<65$ years. A total of $75.8 \%$ of younger female participants with refractory disease experienced at least one exacerbation, and $69.7 \%$ had experienced an ICU admission. For older female participants with refractory disease, $60.6 \%$ and $54.6 \%$ had experienced at least one exacerbation or ICU admission, respectively (between-group differences were not significant; $p=0.19$ and $p=$ 0.21 , respectively). A significantly higher proportion of younger female participants had ever required a feeding tube (30.3\% vs. $6.1 \%$; $p=0.01$ ). In addition to age, gender has previously been shown to impact the clinical course of MG ${ }^{6,25}$ However, the small number of male participants with refractory MG in this study made it impossible to analyze the effect of age on HRU in males with MG.

This study did not examine the costs associated with treating MG, or the cost implications of refractory MG compared with nonrefractory MG. A US medical claims database review of 1,288 patients with MG found that the cost of treating MG was much higher than the cost of treating diseases such as multiple sclerosis, migraine, and Alzheimer's disease. ${ }^{29} \mathrm{Al}-$ though further research is needed into the difference in the costs of treating refractory and nonrefractory MG, the increased HRU of patients with refractory MG suggests higher associated costs.

Some limitations of the present analyses should be noted. The data collected in the MGFA Patient Registry are self-reported, and clinical diagnoses and documented therapies were not confirmed by a healthcare professional. Some par- 

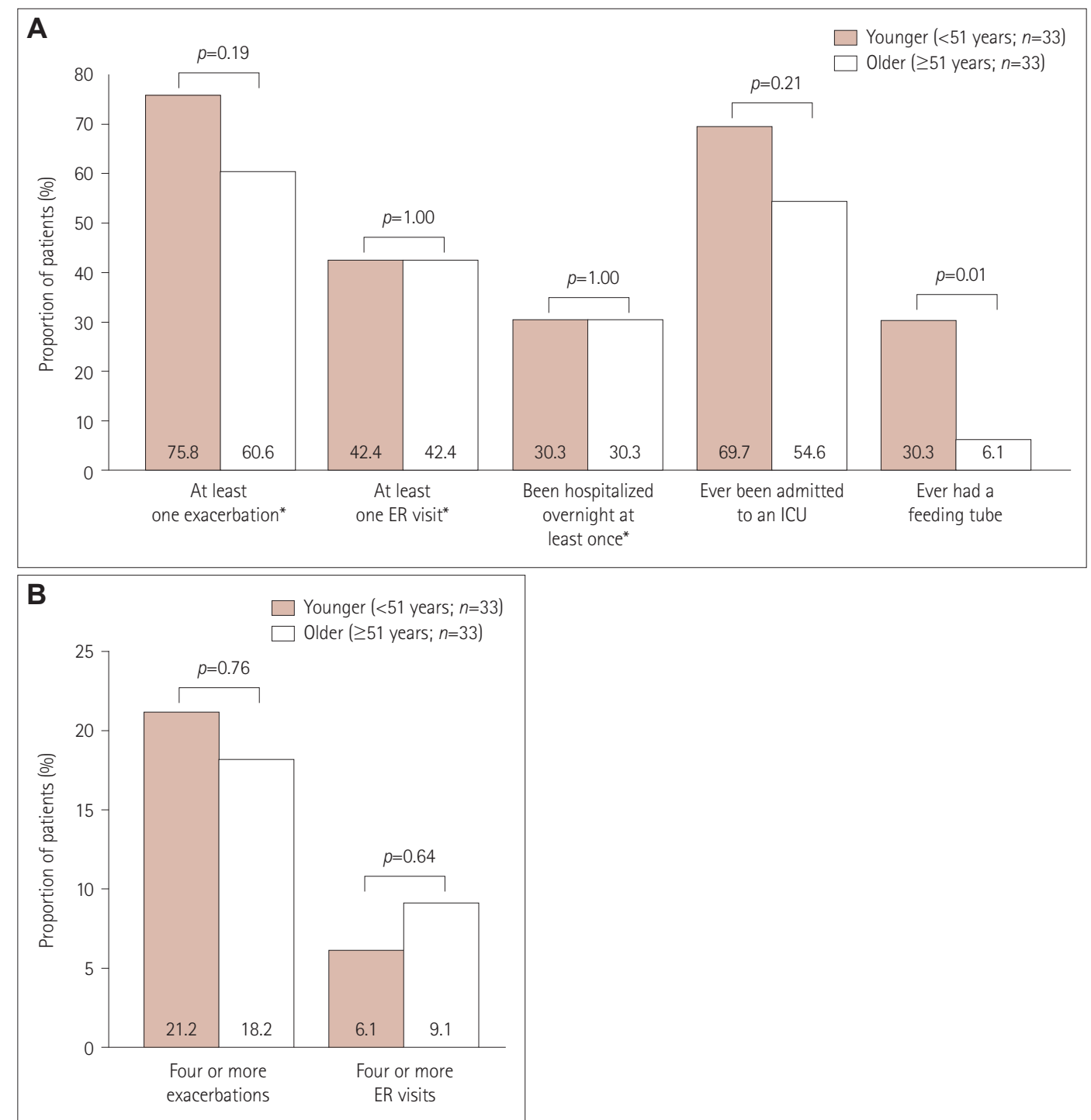

Fig. 5. Exacerbations in and healthcare resource utilization by younger and older female participants with refractory MG (A) and numbers of exacerbations and ER visits during the 6 months before enrollment in younger and older female participants with refractory MG (B). Frequencies of study variables were compared between participants with refractory and nonrefractory MG using $\chi^{2}$ tests for categorical variables and the number of exacerbations, and using Fisher's exact test for the number of ER visits. $p$ values are for comparisons between the refractory- and nonrefractory-MG groups, and were calculated after excluding data from participants with unknown/missing data. *During the 6 months before enrollment. ER: emergency room, ICU: intensive care unit, MG: myasthenia gravis.

ticipants with uncontrolled MG might not have met all of the study-specific criteria for having refractory disease. The inclusion of such participants in the nonrefractory-MG group would have reduced the magnitude of differences observed between the refractory- and nonrefractory-MG groups. Conversely, we acknowledge that some of the participants who met the criteria for refractory disease had uncontrolled disease for reasons such as inadequate treatment dose, short time receiving their current treatment, or poor treatment compliance.

Participants enrolled in the MGFA Patient Registry might constitute a self-selected sample of participants with MG who are more inclined to seek medical information, or who have a higher education level, higher socioeconomic status, and better Internet access, thereby facilitating their participation. This may be reflected in the characteristics of the participants in the MGFA Patient Registry, notably the high proportion of females, who are in general more likely than males to access healthcare, ${ }^{30}$ and the differing ethnicity distribution compared with the general MG population in the USA. It is also possible that patients with refractory MG are more motivated to participate in the registry, which may have resulted in the proportion of participants with refractory disease in this study being higher than that in the general MG population. Such self-selection may reduce the generalizability of these 
findings to the wider MG population.

Participants were asked to provide the total number of times that they had visited an ER during the 6 months before enrollment, with no instruction to include only ER visits for reasons relating to their MG. In addition, participants were given the option to select the reason for any overnight hospitalizations, which included exacerbation or worsening of MG, rehabilitation, other MG-related problem, or non-MG-related problems. The inclusion of ER visits and overnight hospitalizations not related to MG might confound the analyses. Although only exacerbations, ER visits, and overnight hospitalizations that had occurred during the 6 months before enrollment were recorded, no time restriction was applied to the history of ICU admission or feeding-tube requirement in the enrollment survey. Future studies should investigate all HRU using the same time frame. Because data regarding age in the refractory group were limited to female participants aged $<65$ years, these analyses might not be generalizable to the wider population of patients with refractory MG. However, this study builds on the growing evidence that patients with refractory MG suffer from a higher disease burden and negative impacts on their daily lives than patients with nonrefractory MG. Further research is required to assess the cost implications of increased HRU due to refractory MG.

In conclusion, this analysis of US participants in the MGFA Patient Registry has highlighted the burden of MG on both patients with refractory and patients with nonrefractory disease. More specifically, this study found that participants with MG that is refractory to traditional therapies experience more disease-related morbidities - which are associated with higher levels of HRU-than participants with nonrefractory MG. There remains a need for therapies that are effective against refractory MG, improve outcomes, and reduce the HRU by these patients.

\section{Conflicts of Interest}

Linda A. Harris is employed by Alexion Pharmaceuticals, Inc.; Gary Cutter, Haichang Xin, and Inmaculada B. Aban are employed by the University of Alabama at Birmingham, which received financial support from Alexion for this study. Gary Cutter is also President of Pythagoras, Inc., a private consulting company located in Birmingham, AL, USA, and is Professor of Biostatistics at the School of Public Health at the University of Alabama at Birmingham. Gary Cutter has served as a member of consulting or advisory boards (Argenx, Atara Biotherapeutics, Axon, Biogen, BrainStorm Cell Therapeutics, Charleston Laboratories, Click Therapeutics, Genentech, Genzyme, GW Pharmaceuticals, Klein Buendel, MedDay Pharmaceuticals, MedImmune, Novartis, Roche, SciFluor Life Sciences, Somahlution, Teva, TG Therapeutics, and UT Houston), and data and safety monitoring boards [AMO Pharma, BioLineRx, Hisun USA, and Horizon Pharma, Merck, Merck/Pfizer, National Heart, Lung, and Blood Institute (NHLBI; Protocol Review Committee), Neurim Pharmaceuticals, National Institute of Child Health and Human Development (NICHD; OPRU Oversight Committee), Novartis, OPKO Biologics, Orphazyme, Reata Pharmaceuticals, Receptos/Celgene, Sanofi-Aventis, and Teva].
Acknowledgements

This study was funded by Alexion Pharmaceuticals. The University of Alabama at Birmingham, Birmingham, AL, USA received financial support from Alexion to provide the raw data, statistical analyses, and data interpretation for the study. The authors would like to thank the Myasthenia Gravis Foundation of America (MGFA) for establishing the Myasthenia Gravis Patient Registry and for its continued support, the MGFA Patient Registry Committee, and the coordinating center at the University of Alabama at Birmingham. The authors would also like to thank Róisín Armstrong, Kelley Capocelli, Kenji Fujita, and Sivani Paskaradevan (Alexion Pharmaceuticals) for critical review of the manuscript. Editorial assistance for this manuscript was provided by Dr Vicky Sanders, Oxford PharmaGenesis, Oxford, UK, with funding from Alexion Pharmaceuticals.

\section{REFERENCES}

1. Heldal AT, Owe JF, Gilhus NE, Romi F. Seropositive myasthenia gravis: a nationwide epidemiologic study. Neurology 2009;73:150-151.

2. Fang F, Sveinsson O, Thormar G, Granqvist M, Askling J, Lundberg IE, et al. The autoimmune spectrum of myasthenia gravis: a Swedish population-based study. J Intern Med 2015;277:594-604.

3. Cetin H, Fülöp G, Zach H, Auff E, Zimprich F. Epidemiology of myasthenia gravis in Austria: rising prevalence in an ageing society. Wien Klin Wochenschr 2012;124:763-768.

4. Park SY, Lee JY, Lim NG, Hong YH. Incidence and prevalence of myasthenia gravis in Korea: a population-based study using the National Health Insurance claims database. J Clin Neurol 2016;12:340-344.

5. Murai H, Yamashita N, Watanabe M, Nomura Y, Motomura M, Yoshikawa $\mathrm{H}$, et al. Characteristics of myasthenia gravis according to onset-age: Japanese nationwide survey. J Neurol Sci 2011;305:97-102.

6. Grob D, Brunner N, Namba T, Pagala M. Lifetime course of myasthenia gravis. Muscle Nerve 2008;37:141-149.

7. Robertson NP, Deans J, Compston DA. Myasthenia gravis: a population based epidemiological study in Cambridgeshire, England. J Neurol Neurosurg Psychiatry 1998;65:492-496.

8. Melzer N, Ruck T, Fuhr P, Gold R, Hohlfeld R, Marx A, et al. Clinical features, pathogenesis, and treatment of myasthenia gravis: a supplement to the Guidelines of the German Neurological Society. J Neurol 2016;263:1473-1494.

9. Zieda A, Ravina K, Glazere I, Pelcere L, Naudina MS, Liepina L, et al. A nationwide epidemiological study of myasthenia gravis in Latvia. Eur J Neurol 2018;25:519-526.

10. Muppidi S, Wolfe GI, Conaway M, Burns TM; MG Composite and MG-QoL15 Study Group. MG-ADL: still a relevant outcome measure. Muscle Nerve 2011;44:727-731.

11. Hoffmann S, Ramm J, Grittner U, Kohler S, Siedler J, Meisel A. Fatigue in myasthenia gravis: risk factors and impact on quality of life. Brain Behav 2016;6:e00538.

12. Paul RH, Cohen RA, Goldstein JM, Gilchrist JM. Fatigue and its impact on patients with myasthenia gravis. Muscle Nerve 2000;23:14021406.

13. Silvestri NJ, Wolfe GI. Treatment-refractory myasthenia gravis. J Clin Neuromuscul Dis 2014;15:167-178.

14. Wendell LC, Levine JM. Myasthenic crisis. Neurohospitalist 2011;1: 16-22.

15. Conti-Fine BM, Milani M, Kaminski HJ. Myasthenia gravis: past, present, and future. J Clin Invest 2006;116:2843-2854.

16. Sanders DB, Wolfe GI, Benatar M, Evoli A, Gilhus NE, Illa I, et al. International consensus guidance for management of myasthenia gravis: executive summary. Neurology 2016;87:419-425.

17. Suh J, Goldstein JM, Nowak RJ. Clinical characteristics of refractory myasthenia gravis patients. Yale J Biol Med 2013;86:255-260.

18. Mantegazza R, Antozzi C. When myasthenia gravis is deemed refrac- 
tibody-positive refractory generalised myasthenia gravis (REGAIN): a phase 3, randomised, double-blind, placebo-controlled, multicentre study. Lancet Neurol 2017;16:976-986.

25. Lee I, Kaminski HJ, Xin H, Cutter G. Gender and quality of life in myasthenia gravis patients from the myasthenia gravis foundation of America registry. Muscle Nerve 2018;58:90-98.

26. Gilhus NE, Nacu A, Andersen JB, Owe JF. Myasthenia gravis and risks for comorbidity. Eur J Neurol 2015;22:17-23.

27. Diaz BC, Flores-Gavilán P, García-Ramos G, Lorenzana-Mendoza NA. Myasthenia gravis and its comorbidities. J Neurol Neurophysiol 2015;6:1-5.

28. Tanovska N, Novotni G, Sazdova-Burneska S, Kuzmanovski I, Boshkovski B, Kondov G, et al. Myasthenia gravis and associated diseases. Open Access Maced J Med Sci 2018;6:472-478.

29. Guptill JT, Marano A, Krueger A, Sanders DB. Cost analysis of myasthenia gravis from a large U.S. insurance database. Muscle Nerve 2011; 44:907-911.

30. Bertakis KD, Azari R, Helms LJ, Callahan EJ, Robbins JA. Gender differences in the utilization of health care services. J Fam Pract 2000; 49:147-152. 